\title{
The inclusion of delirium in version 2 of the National Early Warning Score will substantially increase the alerts for escalating levels of care: findings from a retrospective database study of emergency medical admissions in two hospitals 辪
}

\author{
Authors: Mohammed A Mohammed, ${ }^{\mathrm{A}}$ Muhammad Faisal, ${ }^{\mathrm{B}}$ Donald Richardson, ${ }^{\mathrm{C}}$ Andy Scally, ${ }^{\mathrm{D}}$ Robin Howes, ${ }^{\mathrm{E}}$ \\ Kevin Beatson, ${ }^{F}$ Sally $\operatorname{Irwin}^{G}$ and Kevin Speed ${ }^{H}$
}

\section{Background}

The National Early Warning Score (NEWS) is being replaced with NEWS2 which adds 3 points for new confusion or delirium. We estimated the impact of adding delirium on the number of medium/high level alerts that are triggers to escalate care.

\section{Methods}

Analysis of emergency medical admissions in two acute hospitals (York Hospital (YH) and Northern Lincolnshire and Goole NHS Foundation Trust hospitals (NH)) in England. Twenty per cent were randomly assigned to have delirium.

Results

The number of emergency admissions (YH: 35584; NH: 35795), mortality (YH: 5.7\%; NH: 5.5\%), index NEWS (YH: 2.5; NH: 2.1) and numbers of NEWS recorded (YH: 879193; NH: 884072) were similar in each hospital. The mean number of patients with medium level alerts per day increased from 55.3 (NEWS) to 69.5 (NEWS2), a $25.7 \%$ increase in $\mathrm{YH}$ and 64.1 (NEWS) to 77.4 (NEWS2), a $20.7 \%$ increase in $\mathrm{NH}$. The mean number of patients with high level alerts per day increased from 27.3 (NEWS) to 34.4 (NEWS2), a $26.0 \%$ increase in $\mathrm{YH}$ and 29.9 (NEWS) to 37.7 (NEWS2), a $26.1 \%$ increase in NH.

\section{Conclusions}

The addition of delirium in NEWS2 will have a substantial increase in medium and high level alerts in hospitalised

\footnotetext{
Authors: Aprofessor, The Strategy Unit, NHS Midlands and Lancashire Commissioning Support Unit, West Midlands, UK and University of Bradford Faculty of Health Studies, Bradford, UK; ${ }^{B}$ senior research fellow, University of Bradford Faculty of Health Studies, Bradford, UK; ' deputy medical director, York Teaching Hospital NHS Foundation Trust, York, UK; D senior lecturer, School of Clinical Therapies, University College Cork, Cork, Ireland; ${ }^{E}$ digital research and development manager, Northern Lincolnshire and Goole Hospitals NHS Foundation Trust, Grimsby, UK; F development manager, York Teaching Hospital NHS Foundation Trust, York, UK; ${ }^{G}$ consultant in elderly care medicine, York Teaching Hospital NHS Foundation Trust, York, UK; ${ }^{H}$ consultant haematologist, Northern Lincolnshire and Goole Hospitals NHS Foundation Trust, Grimsby, UK
}

emergency medical patients. Rigorous evaluation of NEWS2 is required before widespread implementation because the extent to which staff can cope with this increase without adverse consequences remains unknown.

KEYWORDS: National Early Warning Score, NEWS2, delirium, alert, escalation

\section{Introduction}

Early warning scores (EWS) are extensive used in hospitals worldwide to facilitate the optimal management of patients at risk of deterioration and subsequent avoidable morbidity and mortality. ${ }^{1,2}$ EWS are designed to rapidly identify deterioration via the patient's vital signs and, where necessary, to trigger a response from medical teams such as critical outreach to manage the deteriorating patient. The successful management of deterioration involves a complex chain of events which commences with EWS and concludes with timely and appropriate medical care. ${ }^{3}$

In NHS hospitals in England, the patient's vital signs are monitored and summarised into a National Early Warning Score (NEWS). ${ }^{4}$ NEWS offers a standardised approach to assessing acute illness and is derived from seven physiological variables or vital signs - respiration rate, oxygen saturations, any supplemental oxygen, temperature, systolic blood pressure, heart rate and level of consciousness (alert, voice, pain, unresponsive; AVPU) - which are routinely collected by nursing staff as an integral part of the process of care. The use of NEWS is relevant because 'Patients die not from their disease but from the disordered physiology caused by the disease. ${ }^{5}$ NEWS ranges from 0 , indicating the lowest severity of illness, to a maximum value of 20.

NEWS was found to outperform 33 other EWS in predicting patients at risk of cardiac arrest, unanticipated intensive care unit admission or death within 24 hours. ${ }^{6}$ A raised NEWS score $(\geq 5)$ also performed well in identifying sepsis and has been demonstrated to have increased sensitivity and specificity compared to the quick sequential organ failure assessment and the systemic inflammatory response syndrome criteria. ${ }^{4}$ Several studies have shown that paper based (N)EWS are not reliable 
whereas electronically collected (N)EWS are highly reliable and accurate. $^{6-9}$ A survey in 2017 found that about two-thirds of NHS hospitals use some form of electronic NEWS (eNEWS). ${ }^{7}$

NEWS was launched by the Royal College of Physicians (RCP) in 2012 and gained widespread interest from across the world, including Europe, India and the USA; and the US Navy. ${ }^{4,8}$ As part of their commitment to continually improve NEWS and based on feedback from users, the RCP published NEWS2 in December 2017. ${ }^{9}$ The rollout of NEWS2 in the NHS is supported by NHS England with financial incentives under the Commissioning for Quality and Innovation (CQUIN) framework, 'NHS England is asking all trusts to move to be using NEWS2 by March 2019 as part of their approach on sepsis and to qualify for the CQUIN payment.' NEWS2 is also endorsed by the National Quality Board, 'the NHS should move to using NEWS2 as the single language of sickness across all conditions and settings'. ${ }^{10}$

The main changes in NEWS2 focus on better identification of patients with sepsis, the use of ' 5 ' as a threshold for urgent escalation, safer use of oxygen supplementation in patients with hypercapnic respiratory failure (although this has been questioned $)^{11}$ and the inclusion of confusion/delirium. This latter change extends the level of consciousness from AVPU to ACVPU, where ' $C$ ' represents new confusion or delirium and is allocated 3 points (the maximum for a single variable) and is justified thus:

Patients with acute illness may develop an acutely altered mental state, manifesting as new confusion, delirium or a Glasgow Coma Scale (GCS) $<15$. This is an important sign of acute clinical deterioration requiring urgent clinical assessment. Acutely altered mentation may occur as a consequence of sepsis, hypoxia, hypotension or metabolic disturbances, either alone or in combination. ${ }^{9}$

Despite the changes of NEWS2, the thresholds for escalating care have remained essentially unchanged from the original NEWS thresholds. Low scores of 1 to 4 'should prompt assessment by a competent registered nurse or equivalent, who should decide whether a change to frequency of clinical monitoring or an escalation of clinical care is required'. Medium scores of 5 to 6 'should prompt an urgent review by a clinician with competencies in the assessment of acute illness - usually a ward-based doctor or acute team nurse, who should urgently decide whether escalation of care to a team with critical care skills is required (ie critical care outreach team)'. A high score of 7+ 'should prompt emergency assessment by a clinical team I critical care outreach team with critical care competencies and usually transfer of the patient to a higher-dependency care area.' 9

Although it is estimated that between 10 to $31 \%$ of patients in hospital have delirium, ${ }^{12}$ the impact on workload of adding 3 points for new confusion or delirium to NEWS2 are not reported. In this paper we estimate the increase from adding delirium to NEWS2 in medium and high level alerts, which are triggers to escalate care from nursing staff to senior medical staff and critical outreach respectively, using data from emergency medical admission in two different hospitals over a 2-year period based on prevalence rates for delirium.

\section{Methods}

\section{Setting \& data}

Our cohorts of emergency medical admissions are from three acute hospitals which are approximately 100 kilometres apart in the
Yorkshire \& Humberside region of England - the Diana, Princess of Wales Hospital ( $n \sim 400$ beds) and Scunthorpe General Hospital ( $n \sim 400$ beds) managed by the Northern Lincolnshire and Goole NHS Foundation Trust (NLAG), and York Hospital (YH; $n \sim 700$ beds), managed by York Teaching Hospitals NHS Foundation Trust. For the purposes of this study, the two acute hospitals in NLAG are combined into a single dataset and collectively referred to as NLAG hospitals (NH). So, in essence, our study is based on data from two hospitals, $\mathrm{NH}$ and $\mathrm{YH}$, which have been exclusively using electronic NEWS scoring since at least 2013 as part of their in-house electronic patient record systems. We selected these hospitals because they had electronic NEWS, which are collected as part of the patients' process of care and were agreeable to the study.

We considered all adult ( $\geq 16$-year-olds) emergency medical admissions, admitted after 01 January 2014 and discharged before 31 December 2015 (730 days), with eNEWS. For each emergency admission, we obtained a pseudonymised patient identifier, patient's age (years), sex (male/female), discharge status (alive/dead), admission and discharge date and time, and eNEWS (including its subcomponents; respiratory rate, temperature, systolic pressure, pulse rate, oxygen saturation, oxygen supplementation and alertness). NEWS ranges from 0 indicating the lowest severity of illness to 19; the maximum NEWS value possible is 20 .

\section{Assignment of delirium and calculation of NEWS2}

We used data from a well conducted delirium prevalence study by Ryan et $a l^{13}$ - because delirium is essentially an acute confusional state which can be measured more precisely than confusion using validated instruments. We randomly assigned $20 \%$ of our cohort of patients with delirium (yes/no) on admission by age group $(<50=4.7 \% ; 50-64=15.1 \% ; 65-79=21.3 \% ; 80+=34.8 \%)$ based on findings from Ryan et al. ${ }^{13}$ The overall $20 \%$ prevalence of delirium in patients in an acute hospital is within the 10 to $31 \%$ range reported in an earlier systematic review. ${ }^{11}$ We further assigned recovery (yes/no) from delirium during a stay in hospital based on data from the systematic review as follows: $39 \%$ recovered within 24 hours of admission, $32 \%$ did not recover (ie were discharged with delirium) and $29 \%$ recovered after 24 hours but before discharge. ${ }^{11}$ For assigning binary labels (eg delirium, recovered in 24 hours, delirium at discharge) we used random assignment from a binomial distribution with the specified probabilities. For delirium that recovered within the hospital stay, we used a uniform distribution to randomly select one of the recorded NEWS to identify this as a point in time at which the delirium is deemed to be resolved for a given patient. We did not consider any correlation between delirium and other variables in NEWS because such data are not available.

\section{Estimate of the number of patients with medium and high level alerts per day}

Once delirium was randomly assigned, we calculated a NEWS2 score by adding 3 points to NEWS if the patient was alert (AVPU) and had delirium. We counted the number of patients with low scores ( 0 to 4 ; ie no alerts), medium level alerts (score $=5$ or 6 ) and high level alerts (score $=7+$ ) under NEWS and compared this with NEWS2 per day after excluding the first 14 and last 14 consecutive days to remove the impact of artificially low counts of patients in hospital per day attributable to 'boundary conditions', because our cohort of emergency admissions was defined to be those admitted 
and discharged within a 2-year time period ( $n=730$ days). The period of 14 days was determined by visual inspection of number of emergency patients per day. One patient could have more than one alert in a 24-hour period. Our summary statistics (mean, sd, min, max) from NEWS2 are pooled estimates from 20 simulation iterations for each hospital.

\section{Results}

\section{Cohort characteristics}

After excluding records with no (electronic) NEWS (YH 2.1\%, 768/36352; NH 3.5\%, 1305/37100), we were left with similar numbers of emergency medical admissions in $\mathrm{YH}(\mathrm{n}=35584)$ and $\mathrm{NH}(\mathrm{n}=35795)$ with a similar age, sex and in-hospital mortality profile (see Table 1). The median length of stay was shorter at $\mathrm{YH}$ (3.1 days) compared to $\mathrm{NH}$ ( 4.0 days). The mean index NEWS was similar in both hospitals (YH 2.5; NH 2.1). The profile of the vital signs (Table 1) that made up the index NEWS was also similar in the two hospitals, although oxygen supplementation was lower in $\mathrm{YH}(11.3 \%)$ compared to $\mathrm{NH}(19.6 \%)$. The vast majority of admissions were recorded to be alert as part of their index NEWS (YH 97.1\%; $\mathrm{NH} 98.1 \%$ ).

Table 1. Profile of emergency medical admissions

in each hospital (York Hospital and Northern

Lincolnshire and Goole NHS Foundation Trust

\section{hospitals)}

Characteristic

Number of emergency admissions

included in study, $\mathrm{n}$

Mean age, years (SD)

Male, n (\%)

In-hospital mortality, $\mathrm{n}$ (\%)

Median length of stay, days (IQR)

Index NEWS values

Mean NEWS, $n$ (SD)

\section{Alertness Levels}

Alert, n (\%)

Voice, n (\%)

Pain, n (\%)

Unconscious, n (\%)

Oxygen supplementation, $\mathrm{n}(\%)$

Mean respiratory rate, breaths per minute (SD)

Mean temperature, ${ }^{\circ} \mathrm{C}$ (SD)

Mean systolic pressure, $\mathrm{mmHg}$ (SD)

Mean diastolic pressure, $\mathrm{mmHg}(\mathrm{SD})$

Mean pulse rate, beats per minute

(SD)

Mean per cent oxygen saturation, $\%$ (SD)

$\mathrm{IQR}=$ interquartile range; $\mathrm{NH}=$ Northern Lincolnshire and Goole NHS Foundation Trust hospitals; $\mathrm{SD}=$ standard deviation; $\mathrm{YH}=$ York Hospital.
Impact of delirium on medium and high level alerts under NEWS2

The number of NEWS recorded in each hospital over the 730 days was $879193(\mathrm{YH})$ and $884072(\mathrm{NH})$. After excluding the first 14 and last 14 consecutive days to mitigate artificially low counts of patients in hospital (see methods), the total number of NEWS over the remaining 702 days reduced to 861009 (YH) and 865045 $(\mathrm{NH})$.

The mean number of NEWS per day ( $n=702$ days) in $\mathrm{YH}$ was 1226.50 (standard deviation $(\mathrm{SD})=107.35$ ) and 1232.258 (SD = 121.97 ) in $\mathrm{NH}$. The mean number of emergency medical patients in hospital per day in $\mathrm{YH}$ was 365.2 (SD = 30.5) compared to 384.3 $(\mathrm{SD}=31.7)$ in $\mathrm{NH}$. Table 2 shows the estimated impact of adding 3 points for delirium to NEWS 2 by randomly assigning $20 \%$ of our emergency medical admissions to having delirium on admission, wherein $39 \%$ recovered within 24 hours of admission, $29 \%$ recovered afterwards but before being discharged and $32 \%$ had persistent symptoms at discharge (see methods for details).

The mean number of patients with medium level alerts per day increased from 55.3 (NEWS) to 69.5 (NEWS2), an increase of 14.2 alerts or $25.7 \%$, in $\mathrm{YH}$ and likewise in $\mathrm{NH}$ from 64.1 (NEWS) to 77.4 (NEWS2), an increase of 13.3 alerts or $20.7 \%$. The mean number of patients with high level alerts per day increased from 27.3 (NEWS) to 34.4 (NEWS2), an increase of 7.1 alerts or $26.0 \%$ in $\mathrm{YH}$ and likewise in $\mathrm{NH}$ from 29.9 (NEWS) to 37.7 (NEWS2), an increase of 7.8 alerts or $26.1 \%$. The increases in medium and high level alerts was accompanied by a $\sim 5 \%$ reduction in numbers of patients with low scores (see Table 2 ) in each hospital.

\section{Discussion}

We used estimates of delirium (20\%) in acute hospital from a well-designed study by Ryan et al to determine the increase in medium and high level alerts using observed (actual) data from two different hospitals focusing on emergency medical patients (who are the focus of the NEWS and who consume the vast majority of acute care resources). ${ }^{13}$ We found that the inclusion of delirium to NEWS2 will have a 21 to $26 \%$ increase in alerts. As far as we are aware, similar data has not yet been published and so we are unable to compare our findings with previous studies. Nonetheless, there are some important limitations (see below) in our modelling approach which merit further consideration. This is especially important because the change from NEWS to NEWS2 constitutes a complex intervention in a complex adaptive system and so empirical data is urgently required to determine the impact of NEWS2 in practice.

\section{Limitations}

Our assessment of the increases in alerts is likely to be underestimated because we focused on delirium (acute confusion), whereas NEWS2 includes confusion and delirium, and in Ryan et al's study, confusion ( $52 \%$ ) was reported to be $\sim 2.5$ times more prevalent than delirium $(20 \%) .{ }^{13}$ Indeed, nursing staff may also have other cues for scoring confusion/delirium - for example, for dementia, especially where corroborating history is not available to confirm the diagnosis of dementia. Nevertheless, it is important to highlight that our study assumes that confusion/ delirium will be identified with the same degree of fidelity as described in Ryan et al's study - lower fidelity of measurement 
Table 2. Comparing number of patients with low, medium and high scores under NEWS vs NEWS2 per day in each hospital (York Hospital and Northern Lincolnshire and Goole NHS Foundation Trust hospitals) based on 702 days of data. Values for NEWS2 are based on pooled estimates from 20 iterations.

\begin{tabular}{|c|c|c|c|c|c|c|}
\hline \multirow{3}{*}{$\begin{array}{l}\text { Characteristic per day } \\
\text { Mean number of patients, } n \text { (SD) [minimum, } \\
\text { maximum] }\end{array}$} & \multirow{2}{*}{\multicolumn{3}{|c|}{$\begin{array}{l}\text { YH } \\
365.2(30.5)[289,463]\end{array}$}} & \multirow{2}{*}{\multicolumn{3}{|c|}{$\begin{array}{l}\text { NH } \\
384.3(31.7)[287,475]\end{array}$}} \\
\hline & & & & & & \\
\hline & NEWS & NEWS2 & $\begin{array}{l}\text { Change } \\
\text { in mean }\end{array}$ & NEWS & NEWS2 & $\begin{array}{l}\text { Change } \\
\text { in mean }\end{array}$ \\
\hline $\begin{array}{l}\text { Mean number of patients with low scores } \\
(0-4), n(S D) \text { [minimum, maximum] }\end{array}$ & $\begin{array}{l}302.8(25.8) \\
{[234,381]}\end{array}$ & $\begin{array}{l}286.2(24.7) \\
{[217.7,361.1]}\end{array}$ & $\begin{array}{l}-16.6 \\
(-5.5 \%)\end{array}$ & $\begin{array}{l}312.1(31.5) \\
{[224,407]}\end{array}$ & $\begin{array}{l}296.0(30.1) \\
{[210.5,386.2]}\end{array}$ & $\begin{array}{l}-16.1 \\
(-5.2 \%)\end{array}$ \\
\hline $\begin{array}{l}\text { Mean number of patients with medium } \\
\text { scores (5-6), n (SD) [minimum, maximum] }\end{array}$ & $\begin{array}{l}55.3(10.2) \\
{[29,105]}\end{array}$ & $\begin{array}{l}69.5(11.3) \\
{[41.0,122.3]}\end{array}$ & $\begin{array}{l}+14.2 \\
(+25.7 \%)\end{array}$ & $\begin{array}{l}64.1(10.5) \\
{[38,99]}\end{array}$ & $\begin{array}{l}77.4(11.2) \\
{[49.0,114.4]}\end{array}$ & $\begin{array}{l}+13.3 \\
(+20.7 \%)\end{array}$ \\
\hline $\begin{array}{l}\text { Mean number of patients with high scores } \\
(7+), n(S D) \text { [minimum, maximum] }\end{array}$ & $\begin{array}{l}27.3(6.5) \\
{[10,56]}\end{array}$ & $\begin{array}{l}34.4(7.4) \\
{[15.6,68.9]}\end{array}$ & $\begin{array}{l}+7.1 \\
(+26.0 \%)\end{array}$ & $\begin{array}{l}29.9(7.5) \\
{[12,52]}\end{array}$ & $\begin{array}{l}37.7(8.2) \\
{[16.7,63.6]}\end{array}$ & $\begin{array}{l}+7.8 \\
(+26.1 \%)\end{array}$ \\
\hline
\end{tabular}

is likely to identify fewer cases and so reduce the number of escalations in practice.

Whilst NEWS/NEWS2 offers guidelines on escalations of care based on medium and high level alerts, our data does not permit us to determine the actual numbers of escalations that will occur because, in practice, the decision to escalate following one or more alerts for a given patient is likely to be more complex, involving a range of factors including clinical judgement, previous alerts, ceilings of care and palliative care. Furthermore, unlike NEWS, a single score of 3 in NEWS2 does not now automatically trigger escalation. The guidance for NEWS2 states:

We recommend that new confusion scores 3 on the NEWS chart, ie a red score for a single score of 3 , indicating that the patient requires urgent assessment.

and continues to explain that:

We now recommend that a single extreme value of 3 does not warrant the same level of alert as a NEW score of 5 or more, but should prompt an urgent review by a clinician (usually a wardbased doctor) to determine the cause and decide whether an escalation of care is required and the frequency of subsequent monitoring.

So, the extent to which escalations actually increase in practice requires empirical evidence which may be forthcoming from hospitals that have switched to NEWS2.

The impact of NEWS2 on nursing workload ${ }^{14}$ is also important to determine because NEWS has been implemented by nurses and a 3 point increase in NEWS2 for confusion or delirium will not only lead to more escalations at medium and higher NEWS, but also increased frequency of observations (for $\sim 5 \%$ of patients per day) for nursing staff without highlighting what nurses (and other clinical staff) may no longer be required to do:

A low score (NEW score 1-4) should prompt assessment by a competent registered nurse who should decide if a change to frequency of clinical monitoring or an escalation of clinical care is required.

Moreover, nurses will be required to identify new confusion or delirium and then use this to uplift the NEWS2 score, but unlike the other variables in NEWS2, new confusion or delirium are known to be more challenging to define, measure and monitor in routine clinical practice which will also influence the actual number of alerts in practice. ${ }^{15,16}$

NEWS2 also separately considers patients with hypercapnic respiratory failure, usually due to chronic obstructive pulmonary disease (COPD), who have clinically recommended oxygen saturation of $88-92 \%$. This was because in some COPD patients the combination of low oxygen saturation and an additional score of 2 (NEWS) for supplemental oxygen was likely to trigger at least a medium-level NEWS alert requiring an urgent review. However in some COPD patients (at initial review) the delivery of oxygen could be inappropriately encouraged by NEWS in attempt to restore oxygen to the normal range. Data from one of our hospitals $(\mathrm{YH})$ suggests that approximately $2 \%$ of adult medical inpatients were likely to be affected by this change in NEWS2.

A NEWS2 of $\geq 5$ is recommended as the trigger for escalation and sepsis screening. ${ }^{9}$ The addition of 3 points for confusion or delirium will also increase the number of patients subject to sepsis screening with possible benefits, but also unintended side effects that include increased exposure to inappropriate use of antibiotics because infection is only one of the multifactorial causes of delirium.

Finally, we have not considered many other aspects of the rollout of NEWS2, such as the costs of training staff, time taken to measure and monitor confusion or delirium, costs of changes to paper and IT based NEWS systems and the extent to which the clinical decision-making processes and clinical behaviours change because of the necessary recalibration that NEWS2 entails - a NEWS2 of 5 may no longer have the same predictive value to clinical staff as a NEWS of 5 .

\section{Policy implications}

NEWS is widely used in NHS hospitals and in hospitals in other countries. In the NHS, the introduction of NEWS2, is based on the success of NEWS, with financial incentives for hospitals to introduce NEWS2. ${ }^{10}$ Any increase in the NEWS profile of the hospital population, especially without adjustments to the thresholds for alerts (which we would not advocate - in the absence of confusion or lower oxygen saturation target a NEWS of 5 is the same as a NEWS2 of 5), would result in an increase in the frequency of nursing observations, escalations to base medical staff and to critical care 
outreach teams. Our analysis shows that the introduction of NEWS2 is likely to have substantial impact on the workload, of clinical staff and could undermine the wellbeing of staff and patients because of the increased workload and unintended adverse effects on the quality/safety of care. It might be argued that although the inclusion of confusion/delirium may increase workload it may also lead to earlier intervention and better outcomes for some patients. Such a theory needs rigorous testing and without establishing the evidence base for adding confusion/delirium and that NEWS2 has increased sensitivity, specificity and positive predictive values than NEWS, we may be at risk of inadvertently undermining the efficiency of our escalation systems rather than improving them. ${ }^{9,17}$ So, like other researchers, we also call for a more careful evaluation of NEWS2, with smaller scale testing, before widespread, financially incentivised, implementation. ${ }^{11,17}$

It is, however, important to note that our findings should not detract from the importance and seriousness of confusion or delirium, especially in elderly hospitalised patients. ${ }^{18,19}$ Nevertheless, with a relatively high prevalence of confusion or delirium amongst the hospital population, the proposed implementation of NEWS2 could swamp clinical staff with alerts that yield lower 'signal to noise' ratios than NEWS because a higher NEWS2 may no longer be as indicative of acute deterioration in physiological response to illness as NEWS. ${ }^{11,12}$ Moreover, the appropriate assessment and management of delirium in hospitalised patients requires skilled staff (eg elderly care teams and mental health teams), whereas NEWS2 generally directs escalation to medical and critical care teams. A compromise policy could be to retain confusion or delirium in the AVPU scale (ACVPU) of NEWS2 but without any uplift to the score, and then linking the presence of confusion or delirium to appropriate screening for and assignment to a delirium pathway. ${ }^{16}$ Another advantage of this policy is that it recognises that confusion may be better assessed alongside delirium using a validated tool. ${ }^{12}$ Meanwhile, in the absence of confusion or delirium, patients should continue to be escalated at the same trigger points for escalation as for NEWS. Of course, this revised policy would also need careful evaluation.

\section{Conclusion}

The addition of confusion or delirium in NEWS2 will have a substantial increase in medium and high level alerts in emergency medical patients in hospital, which has the potential to overwhelm the capacity of clinical staff to cope with this increase in workload. Further rigorous evaluation of NEWS2 is required to provide evidence of its benefits before widespread implementation, because the extent to which clinical staff can cope with this increase, without any adverse unintended consequences for staff wellbeing and the quality and safety of care, remains largely unknown.

\section{Funding}

This research was supported by the Health Foundation. The Health Foundation is an independent charity working to improve the quality of healthcare in the UK. This research was supported by the National Institute for Health Research (NIHR) Yorkshire and Humber Patient Safety Translational Research Centre. The views expressed in this article are those of the author(s) and not necessarily those of the NHS, the Health Foundation, the NIHR, or the Department of Health.

\section{Role of the funding source}

The funders of the study had no role in study design, data collection, data analysis, data interpretation, or writing of the report.

\section{References}

1 Downey CL, Tahir W, Randell R, Brown JM, Jayne DG. Strengths and limitations of early warning scores: A systematic review and narrative synthesis. Int J Nurs Stud 2017;76:106-19.

2 Smith MEB, Chiovaro JC, O'Neil M et al. Early warning system scores for clinical deterioration in hospitalized patients: A systematic review. Ann Am Thorac Soc 2014;11:1454-65.

3 Smith G. In-hospital cardiac arrest: Is it time for an in-hospital 'chain of prevention'? Resuscitation 2010;81:1209-11.

4 Royal College of Physicians. National Early Warning Score (NEWS): Standardising the assessment of acute illness severity in the NHS. Report of a working party. London: RCP, 2012.

5 McGinley A, Pearse RM. A national early warning score for acutely ill patients. BMJ 2012;345:e5310.

6 Smith GB, Prytherch DR, Schmidt PE, Featherstone PI. Review and performance evaluation of aggregate weighted 'track and trigger' systems. Resuscitation 2008;77:170-9.

7 NHS Improvement. NEWS baseline survey. PSMU, 2017. http:// psmu.improvement.nhs.uk/workstreams/deterioration/news-baselinesurvey [Accessed September 2018].

8 Royal College of Physicians. NHS England approves use of National Early Warning Score (NEWS) 2 to improve detection of acutely ill patients. London: RCP, 2017. www.rcplondon.ac.uk/news/nhsengland-approves-use-national-early-warning-score-news-2-improvedetection-acutely-ill [Accessed May 2018].

9 Royal College of Physicians: National Early Warning Score (NEWS) 2: Standardising the assessment of acute illness severity in the NHS. Updated report of a working party. London: RCP, 2017.

10 Clark CI. Here's the NEWS: great progress on sepsis but still more to do. NHS England, 2018. www.england.nhs.uk/blog/heres-the-newsgreat-progress-on-sepsis-but-still-more-to-do [Accessed May 2018].

11 Hodgson LE, Congleton J, Venn R, Forni LG, Roderick PJ. NEWS 2 too little evidence to implement? Clin Med 2018;18:371-3.

12 Siddiqi N, House AO, Holmes JD. Occurrence and outcome of delirium in medical inpatients: a systematic literature review. Age and Ageing 2006;35:350-64.

13 Ryan DJ, O'Regan NA, Ó Caoimh R et al. Delirium in an adult acute hospital population: predictors, prevalence and detection. BMJ Open 2013;3:e001772.

14 Mok WQ, Wang W, Liaw SY. Vital signs monitoring to detect patient deterioration: An integrative literature review. Int ] Nurs Pract 2015;21(Suppl 2):91-8.

15 Johnson MH. Assessing confused patients. J Neurol Neurosurg Psychiatry 2001;71(Suppl 1):i7-12.

16 Aird T, McIntosh M. Nursing tools and strategies to assess cognition and confusion. $\mathrm{Br}$ ] Nurs 2004;13:621-6.

17 Pimentel MAF, Redfern OC, Gerry S et al. A comparison of the ability of the National Early Warning Score and the National Early Warning Score 2 to identify patients at risk of in-hospital mortality: a multi-centre database study. Resuscitation 2019;134:147-56.

18 National Institute for Health and Care Excellence. NICE clinical guidelines CG103. Delirium: diagnosis, prevention and management. NICE, 2010.

19 Collier R. Hospital-induced delirium hits hard. Can Med Assoc J 2012;184:23-4.

Address for correspondence: Prof Mohammed A Mohammed, University of Bradford Faculty of Health Studies, Bradford, West Yorkshire BD7 2TT, UK.

Email: m.a.mohammed5@bradford.ac.uk 